\title{
Preparation of a Microporous Polyurethane Film with Negative Surface Charge for siRNA Delivery via Stent
}

\author{
Il-Hoon Cho' and Sangsoo Park ${ }^{2}$ \\ ${ }^{1}$ Department of Biomedical Laboratory Science, College of Health Science, Eulji University, Seongnam, Republic of Korea \\ ${ }^{2}$ Department of Biomedical Engineering, College of Health Science, Eulji University, 553 Sansungdai-ro, Soojung-gu, \\ Seongnam, Republic of Korea \\ Correspondence should be addressed to Sangsoo Park; spark@eulji.ac.kr
}

Received 6 November 2016; Accepted 7 February 2017; Published 6 March 2017

Academic Editor: Binnur A. Temel

Copyright (C) 2017 Il-Hoon Cho and Sangsoo Park. This is an open access article distributed under the Creative Commons Attribution License, which permits unrestricted use, distribution, and reproduction in any medium, provided the original work is properly cited.

\begin{abstract}
Polyurethane (PU) and polyethylene glycol (PEG) were used to prepare a porous stent-covering material for the controlled delivery of small interfering RNA (siRNA). Microporous polymer films were prepared using a blend of polyurethane and watersoluble polyethylene glycol by the solution casting method; the PEG component was extracted in water to make the film microporous. This film was dipped in $2 \%$ poly(methyl methacrylate-co-methacrylic acid) solution to coat the polymer film with the anionic polyelectrolyte. The chemical components of the film surface were characterized by Fourier Transform Infrared (FTIR) spectroscopy and its structural morphology was examined by scanning electron microscopy (SEM). The effect of the negatively charged surface after attachment of a fluorescein isothiocyanate- (FITC-) labeled siRNA-polyethyleneimine complex onto the microporous polyurethane film and the controlled release of the complex from the film was investigated by fluorescence microscopy. Fluorescence microscopy showed the PU surface with intense fluorescence by the aggregates of the FITC-labeledsiRNA-PEI complex (measuring up to few microns in size); additionally, the negatively charged PU surface revealed broad and diffuse fluorescence. These results suggest that the construction of negatively charged microporous polyurethane films is feasible and could be applied for enhancing the efficiency of siRNA delivery via a stent-covering polyurethane film.
\end{abstract}

\section{Introduction}

Stents are little mesh tubes used for the treatment of either narrowed arteries or nonvascular lumen of the gastrointestinal, upper and lower respiratory, and urinary tract to alleviate the symptoms caused by the stenosis [1-11]. However, the stenting procedure is invasive to the tissue surrounding the artery or to the nonvascular internal tracts, and tissue hyperplasia is induced because of this invasive procedure. To prevent excessive tissue hyperplasia and the resulting reobstruction of the stented lumen, localized drug delivery via the stent has been attempted, and many antiproliferative drugs, including sirolimus and paclitaxel, are proven effective for lowering the restenosis rate of the procedure. In addition to antiproliferative drugs, some small interfering RNAs (siRNAs) have been effectively employed to suppress tissue hyperplasia after stenting [12-15]. siRNAs can be incorporated into a stent-covering for localized siRNA delivery to the tissue around the stent. Various methods to incorporate siRNA into the stent surface have been developed, because siRNA is a negatively charged polymer and can bind to cationic polymers or a cationized surface through Coulombic interactions [16-18]. The negatively charged siRNA can be incorporated into cationic polymers such as poly-L-lysine, polyethyleneimine (PEI), or chitosan to form a complex, which facilitates siRNA uptake by a cell. Aktl siRNA-PEI complexes were reportedly immobilized on a hyaluronic acid-coated stent surface, and after treatment with these complexes in the rat VSMC line, suppression of Aktl protein and the downstream signaling proteins that regulate cellular proliferation was observed $[17,18]$.

Effective delivery of siRNA via stent could be achieved, if the stent-covering polymer films were microporous with 
a larger surface area, allowing attachment of more siRNAcationic polyelectrolyte complexes onto the stent-covering. In this study, we developed a microporous polyurethane coating with a negative surface charge to investigate whether the increased polymer film surface area and a negatively charged polymer film surface could enhance the adsorption of siRNAcationic polyelectrolyte complexes onto the stent-covering polyurethane film.

\section{Experimental}

2.1. Materials. Polycarbonate urethane (PU), Chronoflex 85A, was purchased from Advansource Biomedical, Inc. (MA, USA). Polyethylene glycol (PEG) with a weightaveraged molecular weight $(\mathrm{Mw})$ of 8,000 , polyethyleneimine (PEI) with an Mw of 25,000, and poly(methyl methacrylateco-methacrylic acid) (P(MMA-co-MAA)) with a MAA content of $14 \%$, Mn of 15,000, and Mw of 34,000 were purchased from Aldrich in Korea (Yongin, South Korea). Fluorescein isothiocyanate-labeled siRNA (FITC-siRNA) was purchased from Bioneer Co. (Daejeon, Korea). PU was purified by a swelling/deswelling process; PEG was purified by dialysis (molecular weight cut-off: 3,500, Spectrum Laboratories Inc.) against distilled water for one day, and P(MMA-co-MAA) was purified by dissolution in acetone followed by reprecipitation in distilled water. Reagent grade dimethylacetamide (DMAC) and tetrahydrofuran (THF) were obtained from Aldrich. Fluorescein-labeled siRNA was obtained from Dharmacon (Chicago, IL).

2.2. PEG-PU Film Preparation. PEG-PU films containing an appropriate amount of PEG were prepared by dissolving both the polymers in a cosolvent system consisting of THF and DMAC. PEG was dissolved in THF at a concentration of $10 \%$ $\mathrm{w} / \mathrm{v}$, and PU was dissolved in DMAC at a concentration of $10 \% \mathrm{w} / \mathrm{v}$ with magnetic stirring for $3 \mathrm{~h}$ at $40^{\circ} \mathrm{C}$. The PEG and PU solutions were mixed at a ratio of $3: 7$ for preparing a 30 PEG-PU film and at a 5:5 ratio for preparing a 50 PEG-PU film. The above solution $(100 \mu \mathrm{L})$ was then transferred and spread onto a cover glass, followed by drying in a chemical hood for $1 \mathrm{~h}$, and in a convection oven at $70^{\circ} \mathrm{C}$ for $24 \mathrm{~h}$, to remove possible residual solvents.

2.3. Selective Dissolution of PEG for Porous PU Films. Porous PU films were produced by immersing the PEG-PU films in water at $37^{\circ} \mathrm{C}$ for $8 \mathrm{~h}$. The films were then transferred into a convection oven and dried at $70^{\circ} \mathrm{C}$ for $24 \mathrm{~h}$. The dried porous PU films were carefully stored in a vacuum desiccator for future use. The thickness and mass of each dried film were measured by means of a caliper (Mitutoyo, Japan) and an analytical balance.

2.4. P(MMA-co-MAA) Copolymer Coating. P(MMA-coMAA) was dissolved in a mixed solvent of ethanol/acetone $(1: 3, \mathrm{v} / \mathrm{v})$ to prepare 0.1 and $2 \mathrm{w} / \mathrm{v} \% \mathrm{P}(\mathrm{MMA}-\mathrm{co}-\mathrm{MAA})$ solutions. The microporous $\mathrm{PU}$ film was dipped in the $\mathrm{P}$ (MMA-co-MAA) solution at $40^{\circ} \mathrm{C}$ for $3 \mathrm{~s}$, followed by drying in an oven at $70^{\circ} \mathrm{C}$ for $24 \mathrm{~h}$.
2.5. Incorporation of siRNA-PEI Complexes onto the Porous PU Film. The FITC-siRNA-PEI complex $(10 \mu \mathrm{M})$ was prepared in RPMI media. The PU films coated with $0.1 \mathrm{w} / \mathrm{v} \% \mathrm{P}$ (MMAco-MAA) for FITC-siRNA-PEI adsorption were cleansed in an RNAse solution, washed with phosphate buffered saline, and transferred onto a culture plate. Opti-MEM medium $(100 \mu \mathrm{L})$ was added to the wells of the culture plate, followed by addition of $200 \mu \mathrm{L}$ of $5 \mu \mathrm{M}$ FITC-siRNA-PEI complex. The plate was covered with an aluminum foil and incubated for $2 \mathrm{~h}$ at room temperature. The films were then transferred to a fresh plate and washed twice with $500 \mu \mathrm{L}$ Opti-MEM.

2.6. Characterization. PU films prepared under various conditions were evaluated by ATR-FTIR (Bio-Rad FTS 3000 $\mathrm{MX})$. Water contact angles for the microporous $\mathrm{PU}$ film before and after coating with P(MMA-co-MAA) were measured by Phoenix-10 (Surface Electro Optics, Inc., Suwon, Korea).

The surface morphologies (top and bottom) and the cross sections of the prepared films were examined by a scanning electron microscope (SEM, Hitachi-4700, Japan). The incorporation of FITC-siRNA-PEI onto the porous PU films was evaluated by a fluorescence microscope (BX-51, Olympus, Japan), and the release of FITC-siRNA-PEI from the microporous PU films was monitored with a spectrofluorometer (Fluoroskan Ascent FL, Thermo Scientific, USA) at an excitation wavelength of $555 \mathrm{~nm}$ and an emission wavelength of $565 \mathrm{~nm}$.

\section{Results}

3.1. PEG-PU Film Preparation. Figure 1 shows the SEM images of a 30 PEG-PU film (containing 30\% of PEG) before and after the PEG extraction process. Before the PEG extraction process, no pores were observed on the top surface of the film (a); however, microcracks are present on the bottom surface facing the cover glass (b), and the cross section shows no pores but the presence of a scalelike structure (c). After PEG extraction, micropores can be seen on the top surface (d), along with micropores and a layered structure on the lower surface (e), and interconnected micropores are observed on the cross section (f). The structured layer observed on the lower surface facing the cover glass could be due to the easy penetration of water, attracted by hydrophilicity of the glass, and the formation of aqueous layers between the PU film and the cover glass.

SEM images of a 50 PEG-PU film (containing $50 \%$ of PEG) before and after the PEG extraction process are shown in Figure 2. Before PEG extraction, aggregates are observed on the top (a), bottom (b), and the cut surfaces. After the PEG extraction process, the aggregates disappear and micropores are observed instead. Thus, the aggregates in the 50 PEG-PU film, observed in Figures 2(a)-2(c), should be composed of PEG, which is phase-separated from the PEGPU solution during the solvent evaporation process. Upon PEG extraction, the film thickness decreased (Figures 2(c) and 2(f)), and the film appeared too weak for the stentcovering application. Therefore, we decided to investigate 


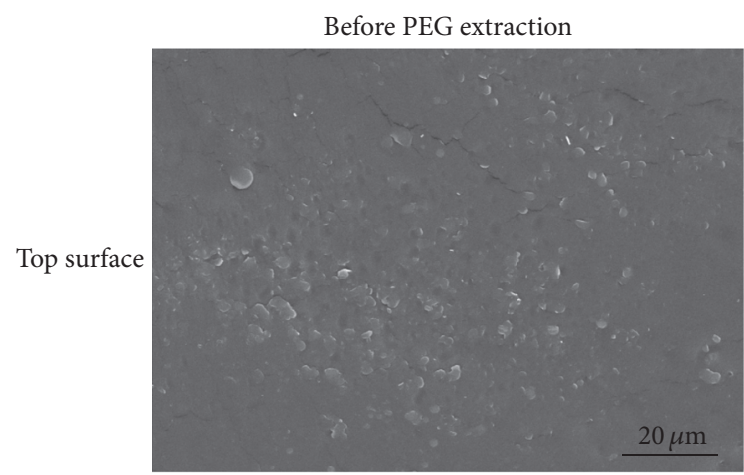

(a)

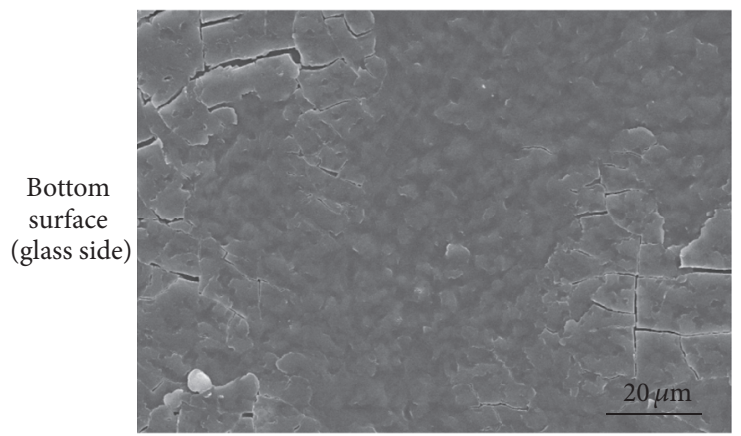

(b)

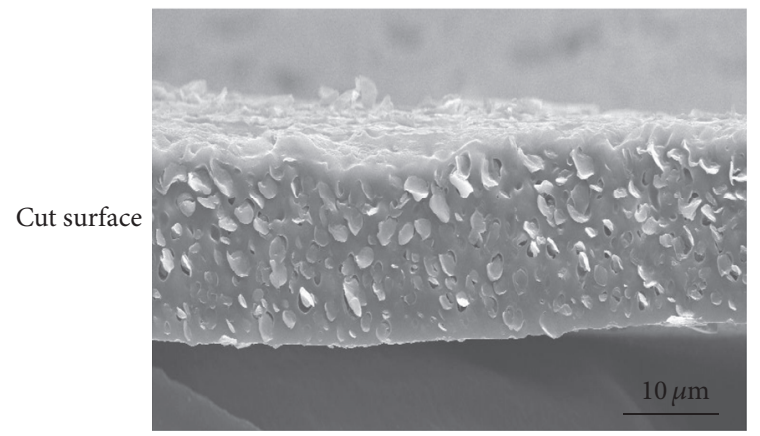

(c)

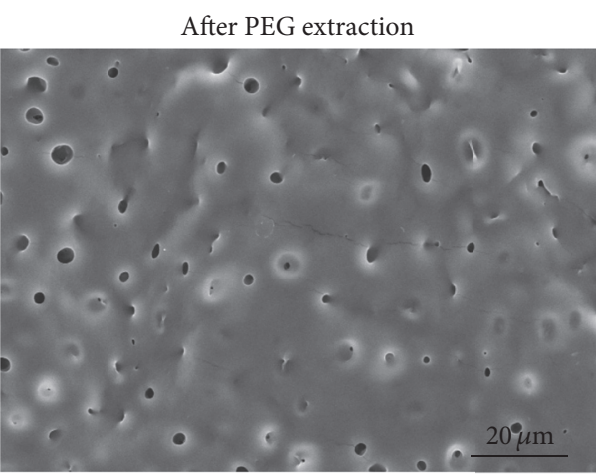

(d)

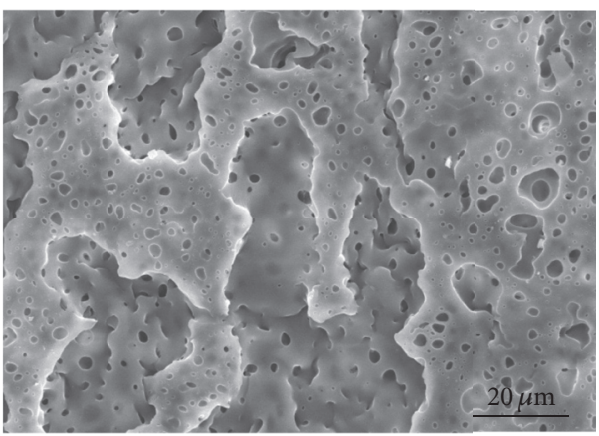

(e)

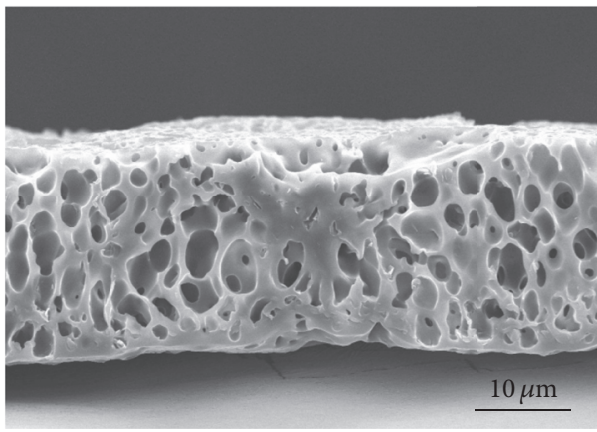

(f)

FIGURE 1: SEM images of 30 PEG-PU films before (a-c) and after PEG extraction (d-f).

only the microporous film prepared from the 30 PEG-PU film for potential stent application.

Figure 3 shows the FTIR spectra of the pure PU film (a) and the 30 PEG-PU film before (b) and after PEG extraction (c). The FTIR spectrum of the pure PU film shows absorption bands at $3480 \mathrm{~cm}^{-1}$ for the secondary amine, at $1725 \mathrm{~cm}^{-1}$ for the amide carbonyl $(\mathrm{C}=\mathrm{O})$, and at $1258 \mathrm{~cm}^{-1}$ for the ether C-O-C group $[19,20]$. As for the 30 PEG-PU composite film, the absorption band at $1110 \mathrm{~cm}^{-1}$ is due to the characteristic absorption of C-O stretching in PEG [20]. After PEG extraction, the characteristic absorption bands of PEG no longer exist, as seen in Figure 3(c), indicating the complete removal of PEG.

3.2. Porous Film Coated with P(MMA-co-MAA). To demonstrate the presence of the $\mathrm{P}(\mathrm{MMA}-\mathrm{co}-\mathrm{MAA})$ layer on the porous film, the P(MMA-co-MAA) coated porous PU film was investigated by ATR-FTIR, and the results are shown in Figure 4.

The ATR-FTIR spectrum of the porous PU film, prepared after PEG extraction from the 30 PEG-PU film as shown in Figure 4(c), is basically the same as that of the PU film, as shown in Figure 3(a). The ATR-FTIR spectrum of $2 \%$ P(MMA-co-MAA) film in Figure 4(b) reveals absorption bands at $1152 \mathrm{~cm}^{-1}$ for $\mathrm{C}-\mathrm{O}$ stretching of the ester group in MMA and MAA, at $1200-1300 \mathrm{~cm}^{-1}$ for the ether C-O$\mathrm{C}$ stretching vibration, and at $1725 \mathrm{~cm}^{-1}$ for the stretching band of the acrylate carboxylate group $(\mathrm{C}=\mathrm{O})$ of $\mathrm{P}(\mathrm{MMA}$ co-MAA) [21]. The ATR-FTIR porous PU film coated with P(MMA-co-MAA) in Figure 4(a) shows a superposition for the $\mathrm{P}$ (MMA-co-MAA) film at $1152 \mathrm{~cm}^{-1}$ and for porous $\mathrm{PU}$ at $1338 \mathrm{~cm}^{-1}$. 


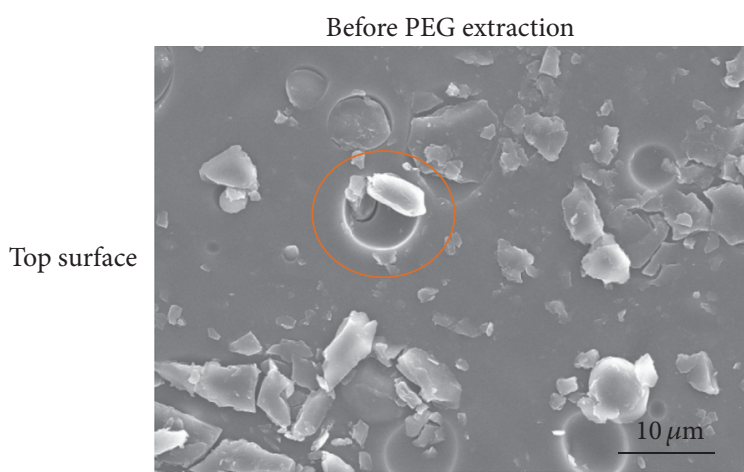

(a)

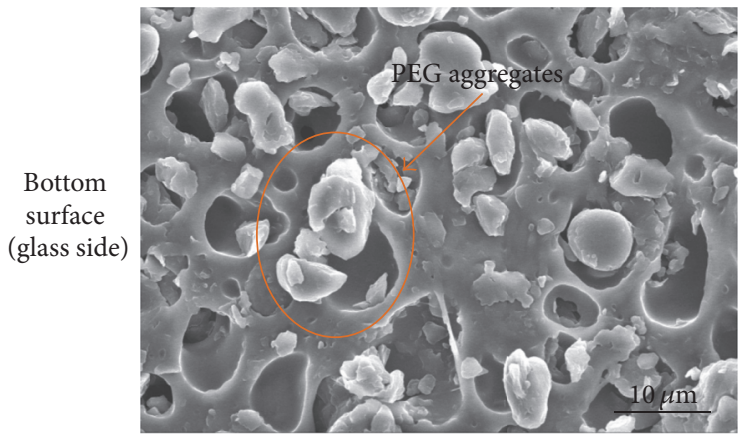

(b)

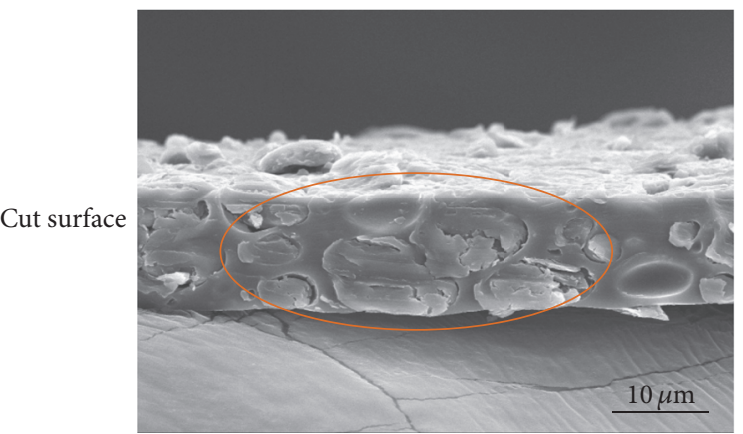

(c)

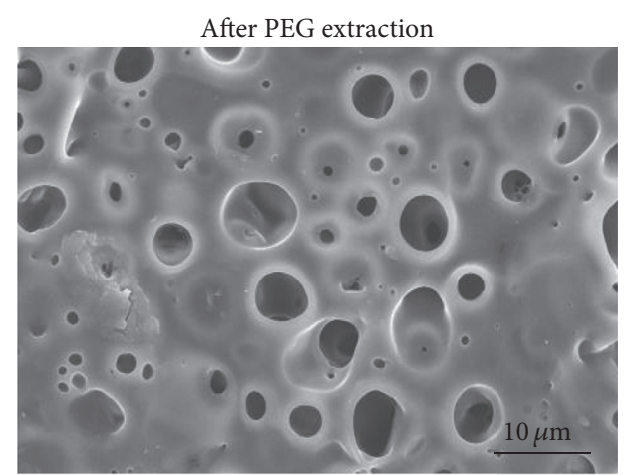

(d)

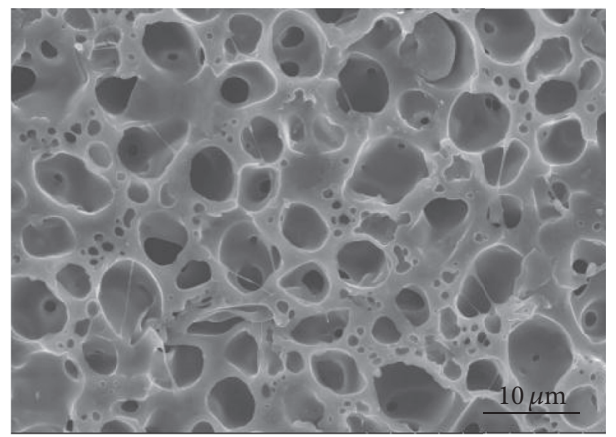

(e)

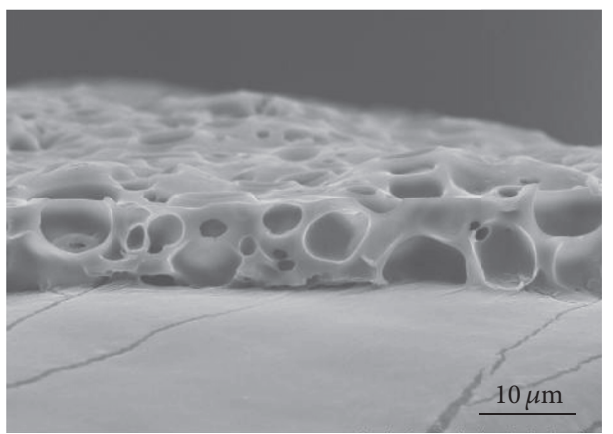

(f)

FIGURE 2: SEM images of the 50 PEG-PU composite film before (a-c) and after (d-f) the PEG extraction process.

3.3. Water Contact Angle. The surface properties of different PU films were evaluated by measuring the water contact angles as shown in Figure 5. The pure PU film surface exhibited a highly hydrophobic characteristic with a water contact angle of $80^{\circ}( \pm 2)$ (a) and this value matched with the unique surface property of pure PU [22]. The contact angle diminished to $58^{\circ}( \pm 1)$, when the PU film was dipped in a $0.1 \% \mathrm{P}(\mathrm{MMA}-\mathrm{co}-\mathrm{MAA})$ solution for $3 \mathrm{~s}$ and dried (b). The contact angle decreased further to $49^{\circ}( \pm 1)$, when the dipping solution was $2 \% \mathrm{P}(\mathrm{MMA}-\mathrm{co}-\mathrm{MAA})(\mathrm{c})$. This indicates that the degree of $\mathrm{P}(\mathrm{MMA}-\mathrm{Co}-\mathrm{MAA})$ coating and the resultant hydrophilicity of the film can be controlled by manipulating the P(MMA-co-MAA) concentration.

3.4. Incorporation of siRNA-PEI Complex on PU Films. To observe the siRNA-PEI complex on the anionic polymer coated PU and pristine PU films, FITC-siRNA-PEI complexes adsorbed onto the PU films were evaluated by fluorescence microscopy. As shown in Figure 6, the FITC-siRNAPEI adsorbed on the PU surface shows aggregates measuring up to few micrometers in size. The aggregates are present on the surfaces of both the PU film coated with P(MMA-coMAA) and the PU film without the coating (b). However, the film coated with P(MMA-co-MAA) exhibits broad fluorescence distribution as well as intense fluorescence from the aggregates. siRNA-PEI aggregates with an average diameter of 80-90 nm have been reported previously by dynamic light scattering [23]. siRNA takes up negative charges; hence, it should be well-soluble in water. However, the negative charge of siRNA is neutralized when it is complexed with the positively charged PEI. The much larger size of the FITCsiRNA-PEI aggregates observed in this study compared to that reported in the above reference could be because of the 

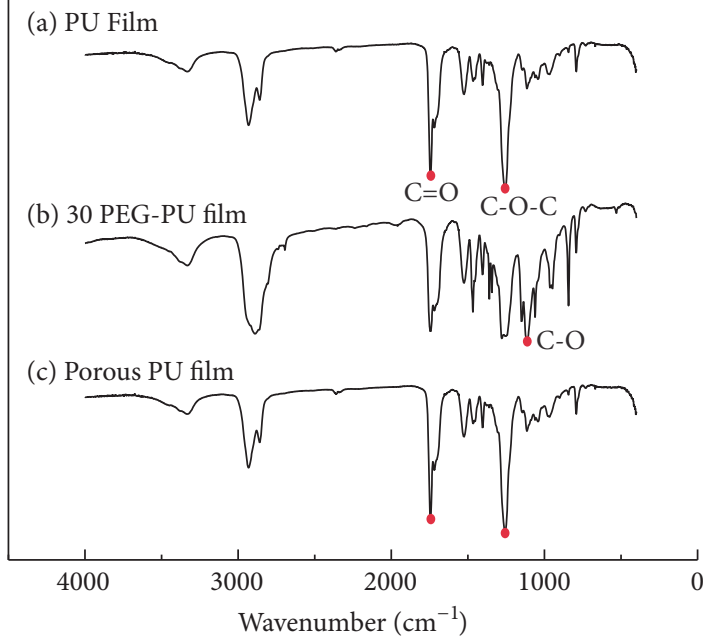

FIGURE 3: FTIR spectra of a pure PU film (a) and a 30 PEG-PU film before (b) and after (c) PEG extraction.

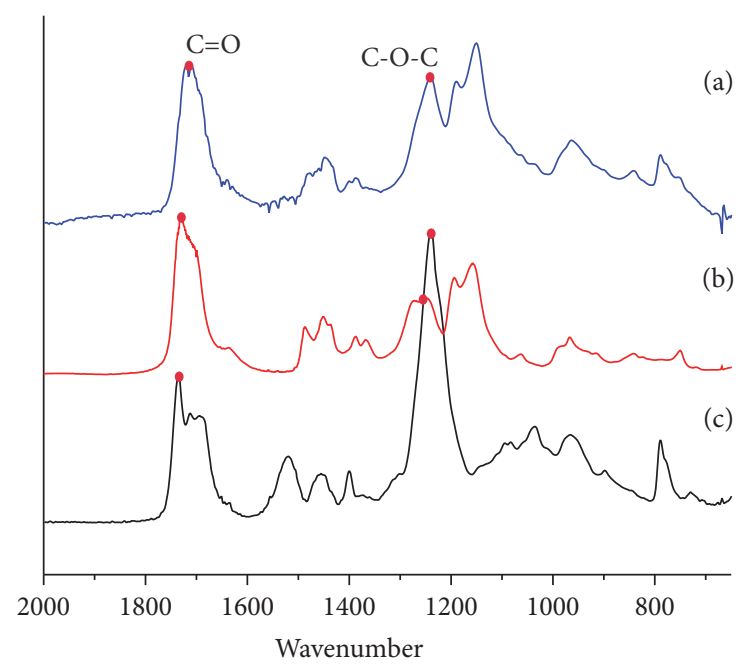

FIGURE 4: FTIR spectra of the P(MMA-co-MAA) coated PU film (a), the 2\% P(MMA-co-MAA) film (b), and the porous PU film (after PEG extraction) (c).

increased hydrophobicity conferred by the FITC label to the siRNA-PEI aggregates.

Intense fluorescence was observed when polymorphonuclear leukocytes including FITC-labeled lipopolysaccharide were aggregated, and a diffuse fluorescence background was observed from isolated and dispersed leukocytes [24]. We interpret the intense fluorescing object as aggregates of the FITC-labeled siRNA-PEI complex and the broad diffuse fluorescing objects as the isolated FITC-labeled siRNA-PEI complexes. Apparently, the concentration of the isolated FITC-labeled-siRNA-PEI complexes increases when the PU surface becomes negatively charged. This could be due to stabilization of the FITC-labeled-siRNA-PEI complexes through Coulombic interactions with the negative charges on the PU surface conferred by the P(MMA-co-MAA) coating.

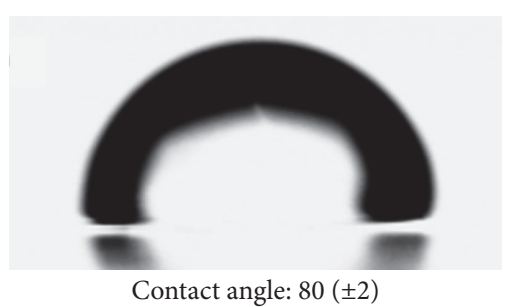

(a)

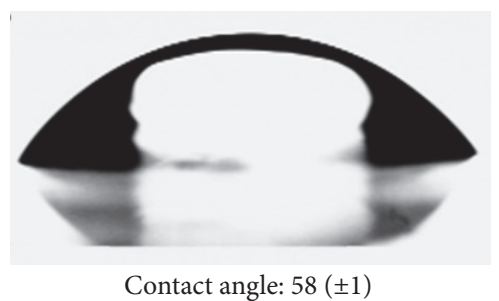

(b)

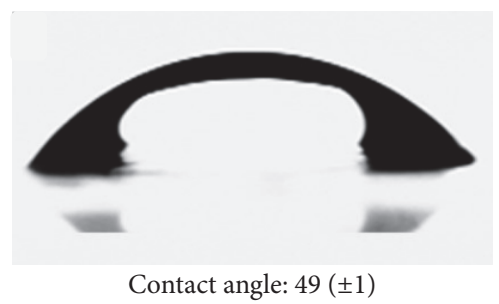

(c)

FIGURE 5: Images and contact angles of water droplets on the porous PU film (a), on a PU film coated with $0.1 \%$ P(MMA-co-MAA) solution (b), and on a PU film coated with $2 \% \mathrm{P}$ (MMA-co-MAA) (c).

3.5. Spectrofluorometry. The cumulative fluorescence intensity of the FITC-labeled siRNA-PEI complex, obtained by spectrofluorometry from the PU film and the anionic polymer treated PU film, is shown in Figure 7. The fluorescence intensity of the FITC-siRNA-PEI complex released from the control PU film was initially around 300, and the intensity increased almost linearly for 2 weeks to around 500 (Figure 7(b)). On the other hand, the intensity of the FITC-siRNA-PEI complex released from the negatively charged porous PU film was initially around 700 and the intensity increased steeply and reached 1,100 (Figure 7(a)). We consider that this increased initial fluorescence intensity from the negatively charged PU surface is because of the aforementioned increased concentration of the FITC-labeled siRNA-PEI complexes.

After the initial burst of release, the release of FITClabeled siRNA-PEI complex is stabilized, and the release process continues for up to 2 weeks, where the steady release rate of the negatively charged porous PU film is approximately 2 times greater than that of the PU film without the coating.

We interpret this slow release process of FITC-labeled siRNA-PEI complexes as being a result of slow decomposition of the aggregates. 


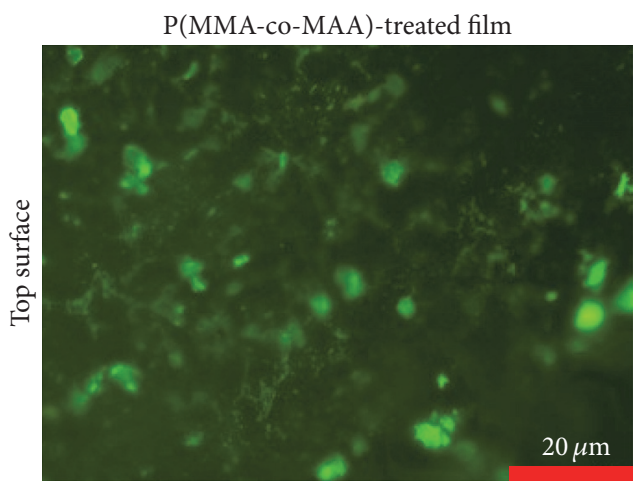

(a)

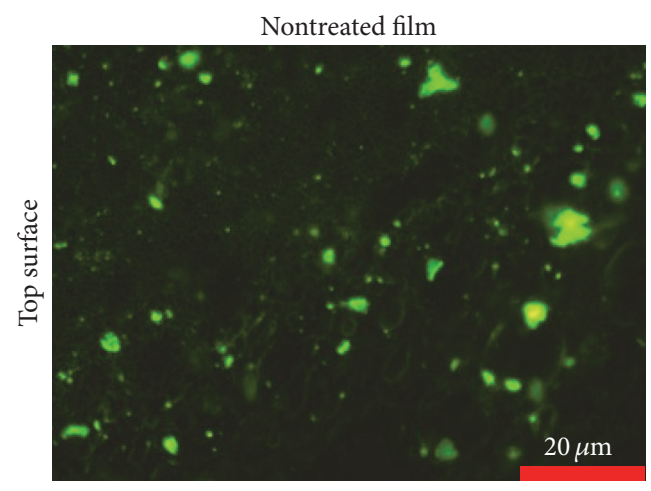

(b)

FIGURE 6: Fluorescence images of the incorporation of siRNA-PEI complex coating on the porous PU films: P(MMA-co-MAA)-treated film (a) and nontreated film (b).

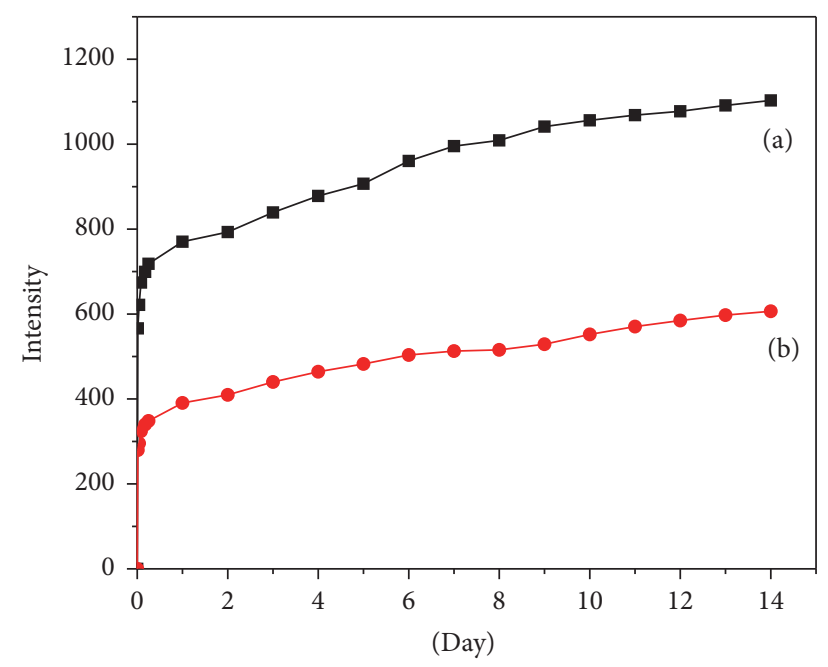

FIGURE 7: Cumulative fluorescence intensities from the microporous films: P(MMA-co-MAA)-treated PU film (a) and nontreated PU film (b).

\section{Conclusions}

In this paper, a microporous polyurethane stent-covering film with negative surface charge and increased surface area was manufactured to enhance the delivery of siRNA-PEI complexes; the microporous film was characterized by SEM, and coating with negatively charged $\mathrm{P}(\mathrm{MMA}-\mathrm{co}-\mathrm{MAA})$ was confirmed by ATR-FTIR and water contact angle measurements. Fluorescence images of the FITC-labeled siRNA-PEI complex on the PU film revealed that siRNA-PEI complexes exist both as aggregates and as separated entities on the negatively charged PU surface. The spectrofluorometry study indicated that the siRNA-PEI complexes dissociate from the surface in two stages: an initial burst stage within an hour and a late steady-state stage for up to 2 weeks. The introduction of anionic charges onto the PU surface appears to enhance both the amount of the adsorbed FITC-labeled siRNA-PEI complexes and their release rate from the PU surface. For clinical application, further studies on the stability of the
siRNA-PEI incorporated PU film during the stent packaging and sterilization processes are warranted.

\section{Competing Interests}

The authors declare that there is no conflict of interests regarding the publication of this paper.

\section{Acknowledgments}

This study was supported by the Korean Ministry of Trade, Industry, and Energy with the National Standard Technical Skill Improvement Project (no. 10049411).

\section{References}

[1] L. Bonello, O. Com, J.-Y. Gaubert, P. Sbraggia, and F. Paganelli, "Covered stent for closure of symptomatic plexus-like coronary fistula," International Journal of Cardiology, vol. 109, no. 3, pp. 408-410, 2006.

[2] A. Bitigen, C. Cevik, B. Turan, and M. Otahbachi, "Use of PTFEcovered stent in acute myocardial infarction of aneurysmatic coronary artery," International Journal of Cardiology, vol. 132, no. 2, pp. e72-e73, 2009.

[3] S. Angeloni, G. Nicolini, F. Nicolao et al., "PTFE-covered stentgrafts for TIPS procedure: one-year patency and clinical results," Journal of Hepatology, vol. 42, pp. 75-76, 2005.

[4] J. W. Lee, S.-G. Yang, and K. Na, "Gemcitabine-releasing polymeric films for covered self-expandable metallic stent in treatment of gastrointestinal cancer," International Journal of Pharmaceutics, vol. 427, no. 2, pp. 276-283, 2012.

[5] S. Moon, S.-G. Yang, and K. Na, "An acetylated polysaccharidePTFE membrane-covered stent for the delivery of gemcitabine for treatment of gastrointestinal cancer and related stenosis," Biomaterials, vol. 32, no. 14, pp. 3603-3610, 2011.

[6] M. J. Chung, H. Kim, K. S. Kim, S. Park, J. B. Chung, and S. W. Park, "Safety evaluation of self-expanding metallic biliary stents eluting gemcitabine in a porcine model," Journal of Gastroenterology and Hepatology, vol. 27, no. 2, pp. 261-267, 2012.

[7] E. Y. Chae, J. H. Shin, H.-Y. Song, J.-H. Kim, T. S. Shim, and D. K. Kim, "Bronchopleural fistula treated with a silicone-covered 
bronchial occlusion stent," Annals of Thoracic Surgery, vol. 89, no. 1, pp. 293-296, 2010.

[8] J. H. Kim, J. H. Shin, H.-Y. Song, S. C. Lee, K. R. Kim, and J.H. Park, "Use of a retrievable metallic stent internally coated with silicone to treat airway obstruction," Journal of Vascular and Interventional Radiology, vol. 19, no. 8, pp. 1208-1214, 2008.

[9] C. J. Yoon, H.-Y. Song, J. H. Kim et al., "Temporary placement of a covered, retrievable, barbed stent for the treatment of hormone-induced benign prostatic hyperplasia: technical feasibility and histologic changes in canine prostates," Journal of Vascular and Interventional Radiology, vol. 21, no. 9, pp. 14291435, 2010.

[10] H. K. Na, H.-Y. Song, H. J. Yeo et al., “Retrospective comparison of internally and externally covered retrievable stent placement for patients with benign urethral strictures caused by traumatic injury," American Journal of Roentgenology, vol. 198, no. 1, pp. W55-W61, 2012.

[11] E.-Y. Kim, J. H. Shin, H.-Y. Song et al., "Suppression of stentinduced tissue hyperplasia in rats by using small interfering RNA to target matrix metalloproteinase-9," Endoscopy, vol. 46, no. 6, pp. 507-512, 2014.

[12] J.-H. Park, J. H. Kim, E.-Y. Kim et al., "Bioreducible polymerdelivered siRNA Targeting MMP-9: suppression of granulation tissue formation after bare metallic stent placement in a rat urethral model," Radiology, vol. 271, no. 1, pp. 87-95, 2014.

[13] E. Kim, H. Song, J. C. Kim et al., "MMP-9 expression after metallic stent placement in patients with colorectal cancer: association with in-stent restenosis," Radiology, vol. 271, no. 3, pp. 901-908, 2014.

[14] M. Shaikh, G. Kichenadasse, N. R. Choudhury, R. Butler, and S. Garg, "Non-vascular drug eluting stents as localized controlled drug delivery platform: preclinical and clinical experience," Journal of Controlled Release, vol. 172, no. 1, pp. 105-117, 2013.

[15] H. J. Kwon and S. Park, "Local delivery of antiproliferative agents via stents," Polymers, vol. 6, no. 3, pp. 755-775, 2014.

[16] H.-L. Che, I.-H. Bae, K. S. Lim et al., "Suppression of postangioplasty restenosis with an Akt1 siRNA-embedded coronary stent in a rabbit model," Biomaterials, vol. 33, no. 33, pp. 85488556, 2012.

[17] C. Choi, J.-P. Nam, and J.-W. Nah, "Application of chitosan and chitosan derivatives as biomaterials," Journal of Industrial and Engineering Chemistry, vol. 33, pp. 1-10, 2016.

[18] A. Szelest-Lewandowska, B. Masiulanis, M. Szymonowicz, S. Pielka, and D. Paluch, "Modified polycarbonate urethane: synthesis, properties and biological investigation in vitro," Journal of Biomedical Materials Research Part A, vol. 82, no. 2, pp. 509520, 2007.

[19] V. Chiono, P. Mozetic, M. Boffito et al., "Polyurethane-based scaffolds for myocardial tissue engineering," Interface Focus, vol. 4, no. 1, 2014.

[20] K. Shameli, M. B. Ahmad, S. D. Jazayeri et al., "Synthesis and characterization of polyethylene glycol mediated silver nanoparticles by the green method," International Journal of Molecular Sciences, vol. 13, no. 6, pp. 6639-6650, 2012.

[21] S. Kiatkamjornwong and S. Tessiri, "Synthesis and characterization of poly[(methyl methacrylate)-co-(methacrylic acid)] for a UV-sensitive aqueous base developable lithographic plate," Journal of Applied Polymer Science, vol. 86, no. 8, pp. 1829-1837, 2002.
[22] M. Khan, J. Yang, C. Shi et al., "Manipulation of polycarbonate urethane bulk properties via incorporated zwitterionic polynorbornene for tissue engineering applications," RSC Advances, vol. 5, no. 15, pp. 11284-11292, 2015.

[23] M. Nourbakhsh, J. Behravan, H. Lage et al., "Nanolipoparticlesmediated MDR1 siRNA delivery: preparation, characterization and cellular uptake," Nanomedicine Journal, vol. 2, no. 1, pp. 3945, 2015.

[24] X. Zhou, X.-P. Gao, J. Fan et al., "LPS activation, of Tolllike receptor 4 signals CD11b/CD18 expression in neutrophils," American Journal of Physiology-Lung Cellular and Molecular Physiology, vol. 288, no. 4, pp. L655-L662, 2005. 

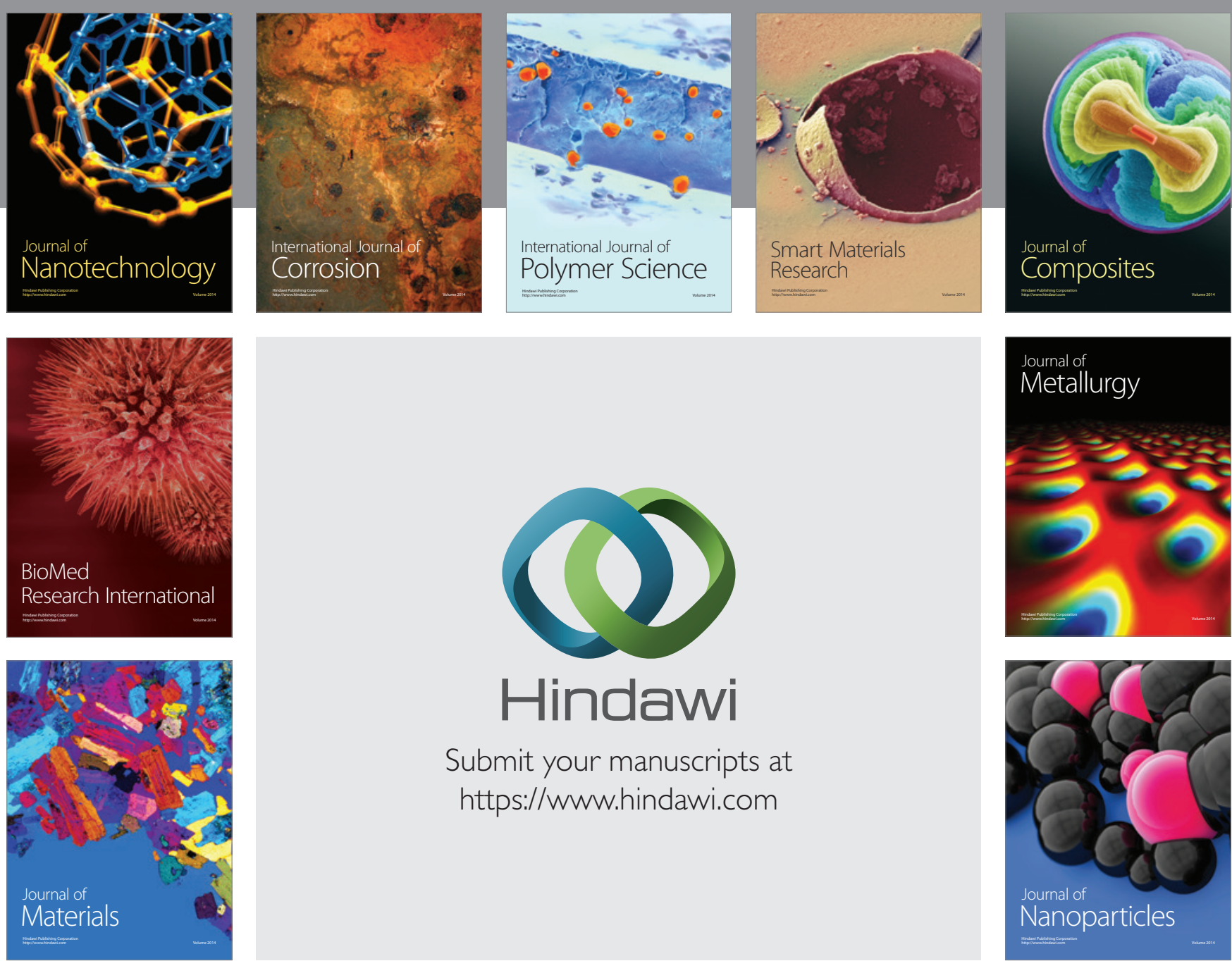

\section{Hindawi}

Submit your manuscripts at

https://www.hindawi.com

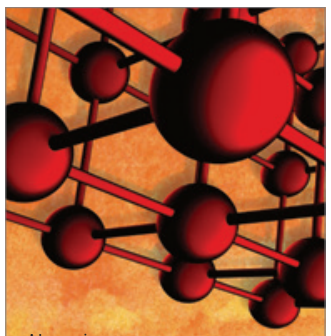

Materials Science and Engineering
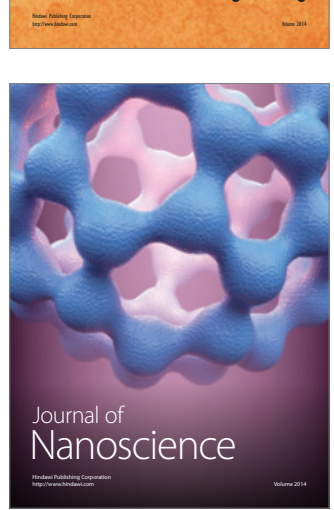
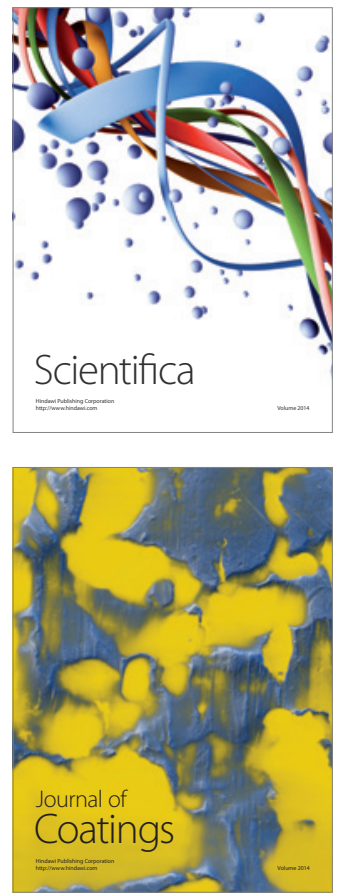
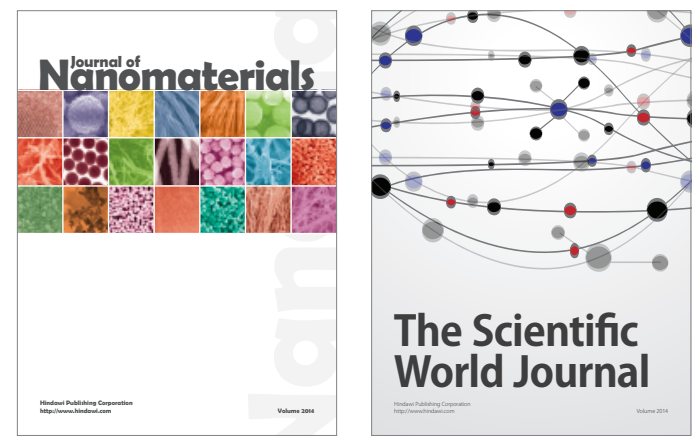

The Scientific World Journal
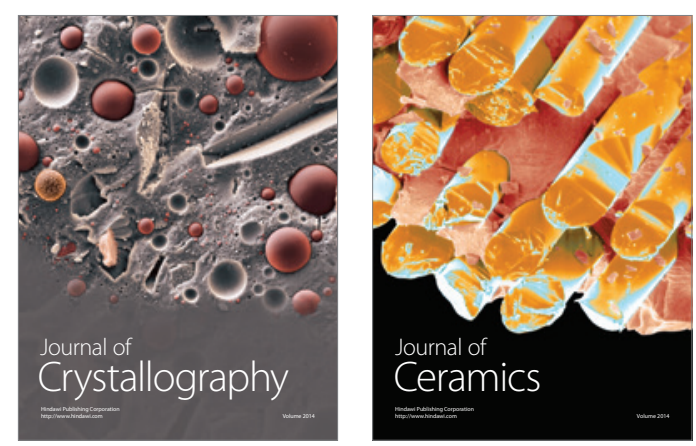
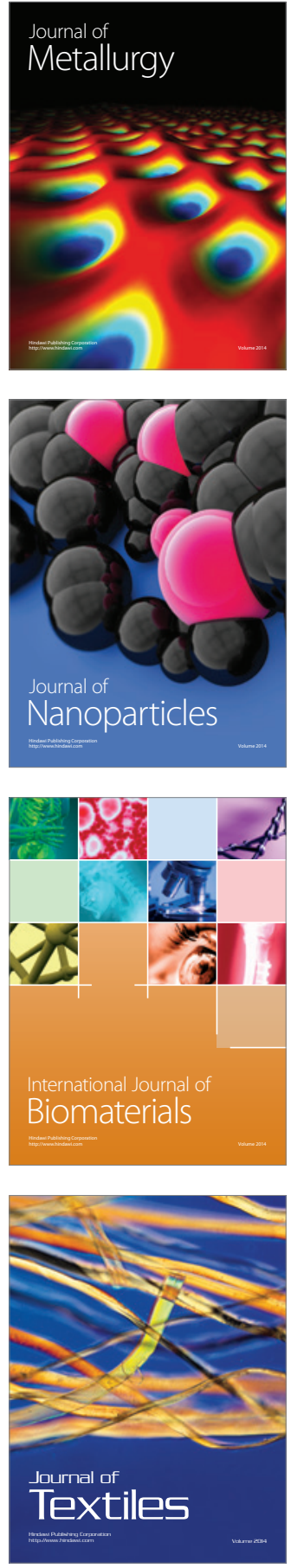